\title{
CARACTERISTICAS CLÍNICAS E CIRURGICAS DE IDOSOS SUBMETIDOS A CIRURGIA CARDÍACA
}

\author{
CLINICAL AND SURGICAL CHARACTERISTICS OF THE ELDERLY \\ SUBMITTED TO CARDIAC SURGERY
}

\author{
André Luiz Cordeiro', André Raimundo Guimarães ${ }^{2}$, Sarah Souza Pontes ${ }^{3}$, Leilane Jesus ${ }^{4}$, \\ Carolina Lima ${ }^{4}$, Vanessa Coutinho ${ }^{4}$
}

\begin{abstract}
Autor para correspondência: André Luiz Cordeiro - andrelisboacordeiro@gmail.com
'Fisioterapeuta. Mestrando em Medicina e Saúde Humana pela Escola Bahiana de Medicina e Saúde Pública. Docente da Faculdade Nobre, Feira de Santana, Bahia, Brasil.

${ }^{2}$ Cirurgião cardíaco do Instituto Nobre de Cardiologia/Santa Casa de Misericórdia, Feira de Santana, Bahia, Brasil. ${ }^{3}$ Fisioterapeuta. Mestre em Desenvolvimento Humano e Responsabilidade Social. Docente da Universidade Federal da Bahia, Salvador, Bahia, Brasil.

${ }^{4}$ Graduanda em Fisioterapia pela Faculdade Nobre, Feira de Santana, Bahia, Brasil.
\end{abstract}

RESUMO | Introdução: A expectativa de vida vem aumentando nas últimas décadas e associado a isso existe também um aumento da incidência de doenças cardiovasculares. Uma das formas de tratamento para essas doenças é a cirurgia cardíaca sendo muito prevalente em idosos. Objetivo: Caracterizar o perfil clínico e cirúrgico de idosos submetidos a cirurgia cardíaca. Metodologia: Foi realizado um estudo retrospectivo observacional com os pacientes internados na Unidade de Terapia Intensiva do Instituto Nobre de Cardiologia. No primeiro dia pós-cirurgia todos os pacientes foram avaliados na busca de dados relacionados ao seu perfil clínico e cirúrgico. Resultados: Durante o período da pesquisa foram operados 51 pacientes, sendo 39 homens $(77 \%)$, com a média de idade mais prevalente acima de 75 anos (30\%), hipertensão arterial e diabetes melitus as comorbidades mais encontradas (67 e 58\%, respectivamente) e 53\% com o Índice de Massa Corpórea dentro da normalidade. Em relação as características cirúrgicas $90 \%$ dos pacientes realizaram cirurgia de revascularização do miocárdio, havendo necessidade em $72 \%$ dos casos de três pontes, tempo de Circulação Extracorpórea de $74 \pm 22$ minutos e tempo de Ventilação Mecânica de $8 \pm 4$ horas. Conclusão: Com base nesses achados pode-se caracterizar que os idosos submetidos a revascularização do miocárdio têm mais de 75 anos, do gênero masculino, hipertensos, e sedentários.

\begin{abstract}
Introduction: Life expectancy has increased in recent decades and associated with this there is also an increased incidence of cardiovascular disease. One form of treatment for these diseases is the heart surgery, which is very prevalent in the elderly. Objective: To characterize the clinical and surgical profile of elderly patients undergoing cardiac surgery. Methods: A retrospective observational study of patients admitted to the Intensive Care Unit of the Nobre Cardiology Institute, in Feira de Santana, Bahia, Brazil. On the first postoperative day, all patients were evaluated in search of data related to their clinical and surgical profile. Results: During the study period 51 patients were operated on, 39 men $(77 \%)$, with an average of more prevalent age above 75 years $(30 \%)$, hypertension and diabetes mellitus being the most frequent comorbidities 167 and $58 \%$, respectively) and $53 \%$ with the body mass index within the normal range. Regarding the surgical characteristics $90 \%$ of patients underwent coronary artery bypass surgery, requiring in $72 \%$ of cases three bridges, extracorporeal circulation time of $74 \pm 22$ minutes and time of mechanical ventilation $8 \pm 4$ hours. Conclusion: Based on these findings, the subjects to myocardial revascularization can be characterized as hypertense, sedentary males over 75 years old.
\end{abstract}

Keywords: Thoracic surgery; Aged; Health Profile. 


\section{INTRODUÇÃO}

Devido ao avanço tecnológico e medicamentoso - Brasil apresenta atualmente uma população com maior expectativa de vida'. Estudos mostram que cerca de $15 \%$ da população do pais são de idosos e associado com essa nova característica da população brasileira encontra-se as mudanças dos hábitos de vida, como sedentarismo e alterações alimentares, o que pode gerar doenças cardíacas ${ }^{1,2}$.

Com o avanço dos anos, o sistema cardiovascular passa por uma série de alterações e estas podem acarretar em doenças cardíacas como insuficiência cardíaca congestiva, doença coronariana, estenose da válvula aórtica, enfraquecimento do músculo cardíaco, doença valvar aórtica devido às alterações degenerativas de fibrose e calcificação valvar.

A cirurgia cardíaca é um procedimento que busca - retorno da capacidade funcional do coração a partir da restauração e restituição das funções vitais. Frequentemente a cirurgia cardíaca é feita para tratar complicações de doença cardíaca isquêmica, corrigir doença cardíaca congênita, ou tratar doença das válvulas cardíacas decorrente de muitas causas incluindo endocardite ${ }^{3}$.

Apesar de a cirurgia cardíaca proporcionar uma melhor qualidade de vida, acarreta em grandes alterações funcionais imediatas nos pacientes, como perda de força muscular e descondicionamento. Mesmo com o processo de modernização dos procedimentos cirúrgicos, existe o comprometimento da função pulmonar, por conta da circulação extracorpórea a perdas funcionais que contribuem há uma limitação do desempenho das atividades de vida diárias ${ }^{4}$.

As características clínicas de pacientes submetidos à cirurgia são de idosos com perfil que apresenta doenças crônicas como hipertensão, diabetes, dislipidemia, tabagistas e com doenças vasculares periféricas, devido a não possuírem desde jovens hábitos de vida saudável ${ }^{5}$. $O$ tratamento cirúrgico deve ser escolhido após a análise de fatores clínicos, funcionais, emocionais e fisiológicos do paciente, visto que com o avanço da idade o risco da cirurgia cardíaca aumenta consideravelmente e agregada a ela há presença comorbidades agravam ainda mais a situação.

Esse trabalho se justifica no intuito de conhecer os pacientes que são submetidos a cirurgia cardíaca e que posteriormente receberão assistência fisioterapêuticas nas unidade hospitalares. Portanto, o objetivo foi descrever o perfil clinico e cirúrgico dos idosos submetidos à cirurgia cardíaca no hospital de referência da cidade de Feira de Santana, Bahia.

\section{MÉTODOS}

Trata-se de um estudo retrospectivo observacional realizado com os pacientes submetidos a cirurgia cardíaca no Instituto Nobre de Cardiologia/Santa Casa de Misericórdia, Feira de Santana - Bahia, no período de Março a Setembro de 2016. O projeto deste estudo foi aprovado pelo Comitê de Ética em Pesquisa da Faculdade Nobre sob o $\mathrm{n}^{\circ} 796580$. Todos os pacientes ou seus responsáveis assinaram o Termo de Consentimento Livre e Esclarecido.

Foram incluídos pacientes com idade acima de 60 anos, de ambos os sexos e submetidos ao procedimento de cirurgia cardíaca (revascularização miocárdica, troca de valvas aórtica e/ou mitral e correção de comunicação interatrial). Como critérios de exclusão, pacientes com dados insuficientes no prontuário, cirurgias associadas e que não aceitem assinar o termo de consentimento livre e esclarecido.

Foram avaliadas as seguintes variáveis: gênero, idade, etnia, hipertensão arterial sistêmica (HAS), Diabetes Mellitus (DM), dislipidemia (DLP), sedentarismo, índice de massa corpórea, tipo de cirurgia, número de pontes, tempo de ventilação mecânica (VM), tempo de circulação extracorpórea (CEC) e caráter da cirurgia.

Dados como HAS, DM e DLP foram coletados através do prontuário médico que foram pesquisados como avaliação pré-operatória. Já as variáveis tipo de cirurgia, número de pontes, caráter da cirurgia e tempo de VM e CEC foram coletados através 
da ficha cirúrgica. O cálculo do IMC obedeceu a fórmula utilizada para a população em geral, sendo o peso (em $\mathrm{kg}$ ) dividido pela altura (em metros) ao quadrado. Para avaliação do nível de atividade física foi utilizado o questionário IPAQ.
Os dados foram armazenados e posteriormente descritos em média e desvio-padrão e as variáveis qualitativas foram expressas em frequências absolutas e relativas.

\section{RESULTADOS}

Foram submetidos a cirurgia cardíaca no período da pesquisa, 60 idosos sendo que 8 foram excluídos por falta de dados no prontuário e 1 devido a cirurgia associada.

Constituída a amostra final de 51 pacientes com predominância do sexo masculino $(77 \%)$, a idade mais prevalente foi acima de 75 anos $(30 \%)$ e etnia parda (53\%). Além disso, a principal comorbidade encontrada foi a HAS $(67 \%)$ e o índice de massa corpórea foi normal (53\%). Todos os valores relativos a características clínicas são expressos na tabela 1.

Tabela 1. Características clínicas dos idosos submetidos a cirurgia cardíaca

\begin{tabular}{|c|c|c|}
\hline & $\mathbf{N}$ & $\%$ \\
\hline $\begin{array}{l}\text { Sexo } \\
\text { Masculino } \\
\text { Feminino }\end{array}$ & $\begin{array}{l}39 \\
12\end{array}$ & $\begin{array}{l}77 \\
23\end{array}$ \\
\hline $\begin{array}{c}\text { Idade (anos) } \\
60-64 \\
65-69 \\
70-74 \\
\text { Acima de } 75\end{array}$ & $\begin{array}{l}17 \\
11 \\
14 \\
18\end{array}$ & $\begin{array}{l}28 \\
18 \\
24 \\
30\end{array}$ \\
\hline $\begin{array}{l}\text { Etnia } \\
\text { Branco } \\
\text { Negro } \\
\text { Pardo }\end{array}$ & $\begin{array}{l}12 \\
12 \\
27\end{array}$ & $\begin{array}{l}24 \\
24 \\
53\end{array}$ \\
\hline $\begin{array}{c}\text { Comorbidades } \\
\text { HAS } \\
\text { DM } \\
\text { DLP } \\
\text { Sedentarismo }\end{array}$ & $\begin{array}{l}32 \\
28 \\
15 \\
41\end{array}$ & $\begin{array}{c}67 \\
58 \\
31 \\
80 \%\end{array}$ \\
\hline $\begin{array}{c}\text { IMC } \\
\text { Eutrófico (Até } 24,9 \mathrm{~m} / \mathrm{kg}^{2} \text { ) } \\
\text { Sobrepeso }\left(25-29,9 \mathrm{~m} / \mathrm{kg}^{2} \text { ) }\right. \\
\text { Obeso (Acima de } 30 \mathrm{~m} / \mathrm{kg}^{2} \text { ) }\end{array}$ & $\begin{array}{c}32 \\
8 \\
20\end{array}$ & $\begin{array}{l}53 \\
13 \\
34\end{array}$ \\
\hline
\end{tabular}

HAS - Hipertensão Arterial Sistêmica; DM - Diabetes Mellitus; DLP - Dislipidemia; IMC - Índice de Massa Corpórea. 
Em relação as características cirúrgicas, o tipo de cirurgia mais prevalente foi a revascularização do miocárdio com 46 pacientes $(90 \%)$, três pontes foram as mais utilizadas $(72 \%)$, o tempo médio de CEC e VM, foram de $74 \pm 22$ minutos e $8 \pm 4$ horas, respectivamente. A tabela 2 evidencia os demais valores.

Tabela 2. Características cirúrgicas dos idosos submetidos a cirurgia cardíaca.

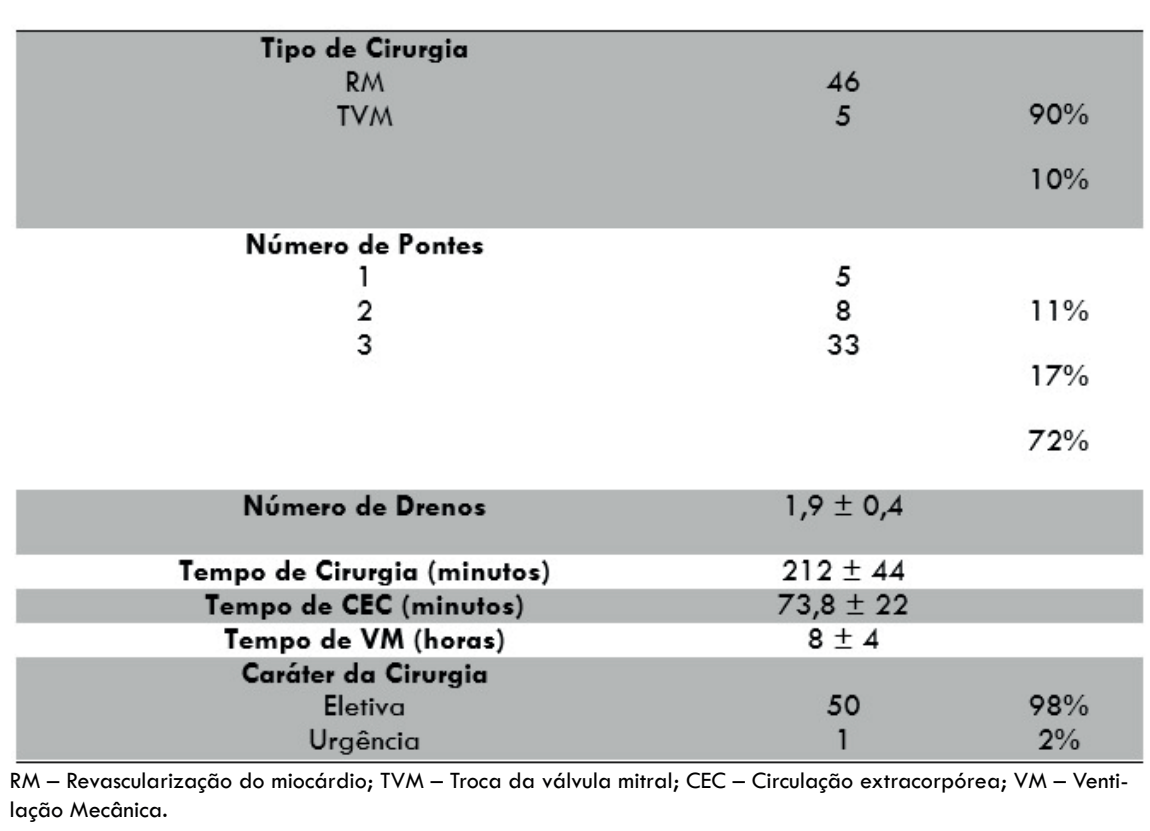

\section{DISCUSSÃO}

Neste estudo, buscou-se descrever as características clínicas e cirúrgicas de idosos submetidos à cirurgia cardíaca, em um Hospital de referência em Feira de Santana, BA. Percebeu-se a existência de uma predominância de pacientes do sexo masculino $(76,9 \%)$ e de idade acima de 75 anos (30\%), submetidos a revascularização do miocárdio e hipertensos.

Quanto ao tempo de VM, foi verificado no presente estudo, uma média de $8 \mathrm{~h}$, esse dado foi inferior ao encontrado em outros estudos, como no de Alexandre et al. ${ }^{6}$ onde a média foi de 26 horas e isso seria justificado pelo fato dos idosos submetidos a cirurgia nessa pesquisa terem idade um pouco mais avançada e normalmente a sua reserva ventilatória ser mais reduzida.

O tempo de CEC visto foi em média de 76 minutos apresentando-se inferior do que em relação ao estudo de Fernandes et al. ${ }^{7}$ onde a média foi de 84,9 minutos, podendo ser devido ao fato dos pacientes apresentarem maiores comorbidades em comparação aos nossos pacientes apesar dos mesmos apresentarem idade superior.

Pacientes idosos apresentam grande dificuldade de serem tratados quando se considera que os riscos para a intervenção cirúrgica são bem maiores que os indivíduos mais novos e sem grandes comorbidades somatizadas. A reserva fisiológica com o avançar da idade se reduz e isso pode resultar em um aumento de doenças que agregadas a comorbidades já existentes, aumenta $\circ$ riscos e a característica da cirurgia.

Todos os pacientes apresentaram algum tipo de comorbidades, foi feita uma comparação com o estudo de Oliveira et al.8 e observou-se que a mesma apresentou alguns percentuais maiores que os do presente estudo, DM $(58,3 \%$ versus $37,2 \%)$, HAS $(66,7 \%$ versus $90,7 \%)$ e dislipidemia $(31,3 \%$ versus $67,4 \%)$, essas doenças agem como fatores predisponentes de doenças cardíacas e que aumentam a necessidade do cuidado e a adoção de condutas específicas ao longo do tempo 
cardiovascular.

Através das nossas pesquisas percebemos que os pacientes que avaliamos com faixa etária acima de 70 anos possuem o IMC normal, sem indicativos de obesidade. Já no estudo de Morsch et al. ${ }^{9}$ a maioria dos seus pacientes com faixa etária abaixo de 65 anos possuíam o IMC alterado, indicativo de sobrepeso, o que também foi visto no artigo de Fernandes et al. ${ }^{7}$ e isto pode estar diretamente ligada a nova geração de idosos que possuíram maus hábitos de vida desde a juventude e que agora apresentam as suas consequências.

Kucewicz-Czech et al. ${ }^{10}$ compararam a taxa de complicação perioperatória entre idosos e pacientes jovens não mostrando diferença entre o tempo de VM porém o tempo de estadia hospitalar e mortalidade foi maior entre os pacientes com idade acima de 75 anos.

Um ponto importante em entender as caraterísticas clinicas e cirúrgicas dos idosos é buscar traçar um protocolo especifico, dentro de cada unidade, para essa população pois sabe-se que associado ao envelhecimento está presente a redução da atividade física ${ }^{11}$, o que foi demonstrado nesse trabalho.

Nesse sentido Van Laar et $a^{12}$. em um estudo com 3.382 pacientes demonstraram que o nível de atividade física é um fator independente para surgimento de complicações pós-operatórias em indivíduos com idade acima de 65 anos.

O estudo foi realizado em apenas um serviço o que pode impactar no poder de generalização dos dados. Perspectivas de ensaios clínicos devem ser incentivados visando o impacto da realização de diferentes níveis de atividade física nessa população em relação a desfechos clínicos e funcionais.
Com base nesses achados pode-se caracterizar que os idosos estudados no hospital de referência de Feira de Santana tem mais de 75 anos, do gênero masculino, hipertensos, sedentários, sendo submetidos a revascularização do miocárdio.

\section{CONTRIBUIÇÕES DOS AUTORES}

Cordeiro ALL participou da concepção e desenho da pesquisa, obtenção de dados, análise e interpretação dos dados, análise estatística e revisão crítica do manuscrito quanto ao conteúdo intelectual. Guimarães AR e Pontes S participaram da revisão crítica do manuscrito quanto ao conteúdo intelectual importante. Santos de Jesus $L$ participou redação e da revisão crítica do manuscrito quanto ao conteúdo intelectual. Lima $C$ participou da redação do manuscrito e da revisão crítica do manuscrito quanto ao conteúdo intelectual. Coutinho $\vee$ participou da redação e da revisão crítica do manuscrito quanto ao conteúdo intelectual.

\section{CONFLITO DE INTERESSES}

Nenhum conflito financeiro, legal ou político envolvendo terceiros (governo, empresas e fundações privadas, etc.) foi declarado para nenhum aspecto do trabalho submetido (incluindo mas não limitandose a subvenções e financiamentos, conselho consultivo, desenho de estudo, preparação de manuscrito, análise estatística, etc).

\section{REFERÊNCIAS}

1. Camargos MC, Gonzaga MR. Viver mais e melhor? Estimativas de expectativa de vida saudável para a população brasileira. Cad. Saúde Pública. 2015;31 (7):14601472. doi: 10.1590/0102-311X00128914

2. Melo TA, Sharp T, Cordeiro ALL. High-intensity strength training in an older population: a preliminary study. Geriatrics, Gerontology and Aging. 2015;9(4):144

3. Santos NP, Mitsunaga RM, Borges DL, Costa MA, Baldez $T E$, Lima IM et al. Factors associated to hypoxemia in patients undergoing coronary artery bypass grafting. Rev Bras Cir Cardiovasc. 2013;28(3):364-70. doi: 10.5935/16789741.20130056

4. Scanlan CL, Wilkins RL, Stoller JK. Fundamentos da terapia respiratória de Egan. 7a ed. Barueri: Manole; 2000

5. Borges DL, Nina VJ, Lima RO, Costa MA, Baldez TE, Santos NP. Características clínicas e demográficas de pacientes submetidos à revascularização do miocárdio em um hospital 
universitário. $2013 ; 14(3): 1$-9

6. Alexandre MC, Nozawa E, Ramos AR, Duarte JV, Battagin AM, Feltrim MI. Caracterização dos octogenários submetidos à cirurgia cardiovascular sob intervenção fisioterapêutica. Revista de Ciência Médica. 201 2;21 (1-6):35-44

7. Fernandes MVB, Aliti G, Souza EM. Perfil de pacientes submetidos à cirurgia de revascularização miocárdica: implicações para o cuidado de enfermagem, Revista Eletrônica de Enfermagem. 2009; 11 (4):993-9

8. Oliveira EL, Westphal GA, Mastroeni MF. Características clínico-demográficas de pacientes submetidos a cirurgia de revascularização do miocárdio e sua relação com a mortalidade Revista Brasileira de Cirurgia Cardiovascular. $2012 ; 27(1): 52-60$. doi: 10.5935/1678-9741.20120009

9. Morsch KT, Leguisamo CP, Camargo M, Coronel W, Mattos CC, Ortiz LDN et al. Perfil ventilatório dos pacientes submetidos a cirurgia de revascularização do miocárdio. Revista Brasileira de Cirurgia Cardiovascular. 2009;24(2): 180-187. doi: 10.1590/S0102-

76382009000200014

10. Kucewicz-Czech E, Kiecak K, Urbańska E, Maciejewski T, Kaliś R, Pakosiewicz W et al. Perioperative care in elderly cardiac surgery patients. Kardiochir Torakochirurgia Pol. 2016;13(4):340-346. doi: 10.5114/kitp.2016.64878

11. Wiegmann B, Ismail I, Haverich A. Cardiac surgery in the elderly. Chirurg. 2017[

12. van Laar C, Timman ST, Noyez L. Decreased physical activity is a predictor for a complicated recovery post cardiac surgery. Health Qual Life Outcomes. 2017 Jan 7;15(1):5. doi: $10.1186 /$ s $12955-016-0576-6$ 